\title{
Estimación del gasto tributario en Cuba
}

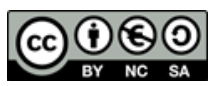

\section{Estimation of tax expenditure in Cuba}

\author{
Elina Martha Díaz López. ${ }^{1}$ \& Caridad Puig Morales. ${ }^{2}$ \\ Recibido: 01-03-2019 / Revisado: 13-03-2019 / Aceptado: 22-03-2019 / Publicado: 05-04-2019
}

Resumen. $\quad$ DOI: https://doi.org/10.33262/concienciadigital.v2i1.952

La investigación realizada aborda el tema del gasto tributario desde una perspectiva teórica y se enmarca en la práctica cubana. Se parte de la teoría aportada por diferentes autores que tratan el tema, para sean comprensibles los términos empleados. Experiencia internacionales fuero un punto de partida en el estudio siempre teniendo en cuenta la coyuntura en que cuba se desarrolla, limitantes y desafíos que enfrenta. Se fundamenta la necesidad de estimar dicho gasto y someterlo a controles similares a los que se somete el gasto directo; buscando la mejora en la calidad de la gestión tributaria. Provee al estado las herramientas para la implementación de la propuesta que se ofrece y hacer así más fácil y completo el trabajo que actualmente se realiza. Se emplearon datos experimentales para demostrar la aplicabilidad del método escogido, las pérdidas de riqueza que enfrentan las actas nacionales y analizar si es realmente factible el modo en que hoy el gobierno maneja este tema.

Palabras claves: tributario, coyuntura, gasto, control, pérdidas

\section{Abstract.}

The research carried out addresses the issue of tax expenditure from a theoretical perspective and is framed in Cuban practice. It is based on the theory contributed by different authors who deal with the subject, so that the terms used are understandable. International experience was a starting point in the study always taking into account the situation in which Cuba develops, limitations and challenges it faces. The need to estimate said expenditure and subject it to controls similar to those that direct expenditure is subjected is based; seeking the improvement in the quality of tax

\footnotetext{
${ }^{1}$ Universidad de La Habana, La Habana, Cuba, emdiaz@gmail.com.

${ }^{2}$ Universidad de La Habana, La Habana, Cuba, cpuig@gmail.com.
} 
management. It provides the state with the tools for the implementation of the proposal that is offered and thus make the work currently being done easier and more complete. Experimental data were used to demonstrate the applicability of the chosen method, the losses of wealth faced by national records and to analyze whether the way in which the government handles this issue is really feasible today.

Keywords: tributary, conjuncture, expense, control, losses.

\section{Introducción.}

El otorgamiento de incentivos o beneficios fiscales en Cuba se sustenta en la propia norma jurídico- tributaria vigente en la actualidad (Ley No. 113 y Decreto No. 308), la cual prevé de acuerdo con los dispuesto en el artículo No. 5 del Libro Primero, "Disposiciones Generales", conceder tres tipos de beneficios; bonificación, condonación y exención. Además, que constituye una práctica de la política fiscal usada por la mayoría de los Estados. Estos incentivos estimulan, en forma de reducciones o exenciones, el pago de ciertos impuestos que se concede a los sujetos pasivos para promover la realización de determinadas actividades consideradas de interés público por el Estado; con la finalidad de promover el desarrollo de actividades y regiones específicas. Estos beneficios adquieren forma a través de dispensa temporales de impuestos y reducción de tasas.

En dependencia del sector se brindan diferentes estímulos; en el caso de los incentivos a la inversión esta: la depreciación acelerada, la deducción parcial, los créditos fiscales. Por otro lado en las zonas especiales con tratamiento tributario privilegiado se encuentran los derechos de importación, el impuesto sobre la renta, el impuesto al valor agregado. Otra forma de estimular es a través de rebajas en impuestos, por la contratación de mano de obra. Otorgando así, beneficios que van desde no pagar un porcentaje de algún impuesto hasta no pagar la totalidad del mismo.

Los incentivos son necesarios aplicarlos en la declaración anual de quien los reciba, para lo cual requerirá asesoría especializada sobre la forma cómo se deben incluir en el formato de declaración anual establecido por la Administración Tributaria.

En las mismas debe aplicar los incentivos fiscales que le correspondan, para de este modo determinar el cálculo de los impuestos que efectivamente le corresponde pagar.

Surge así el problema científico: ¿Cómo estimar el gasto tributario en Cuba?

Partiendo de lo anterior, el objetivo general es proponer la metodología para estimar el gasto tributario en Cuba. Para el cumplimiento de este objetivo, se cumplirán los siguientes objetivos específicos: 
- Definir teóricamente los principales conceptos relacionados con el gasto tributario

- Diseñar la estructura para el Informe de los Presupuestos del gasto tributario en Cuba.

La hipótesis de trabajo: estimar el gasto tributario en Cuba será posible mediante el método ex post.

Para la realización de esta investigación se utilizó como métodos los siguientes:

- Revisión bibliográfica

- Procesamiento estadístico de datos

Con el fin de desarrollar de forma exitosa la presente investigación, primeramente se ofrece una breve descripción acerca de las bases conceptuales y seguidamente se presenta una propuesta del Informe del Presupuesto de gasto tributario y los cálculos que lo complementan.

\section{Desarrollo}

\section{Marco teórico conceptual}

Un sistema tributario regula la relación existente entre las personas naturales, jurídicas y el Estado, donde los dos primeros tienen la obligación de ceder a este último una parte de sus bienes con el objetivo de contribuir al financiamiento de los bienes y servicios públicos. Para hacer efectivo este proceso impositivo de las personas físicas y jurídicas de forma proporcional y equitativa; la Administración Tributaria tiene la responsabilidad de aplicar la legislación fiscal para facilitar e incentivar el cumplimiento voluntario, así como, generar y proporcionar la información necesaria para el diseño y evaluación de la política tributaria ( Sarduy, 2011).

Cuando se logra percibir lo máximo posible utilizando las leyes vigentes estamos en presencia de la recaudación potencial, sujeta a las brechas políticas y otras variantes que aminoran la recaudación; generando Gastos Tributarios que afianzan la brecha mencionada.

Cuando el Estado percibe gastos tributarios es porque exime a determinadas personas naturales o jurídicas del pago del impuesto; ofreciendo así beneficios fiscales. Estos no son más que ventajas estatales donde determinadas empresas o autónomos reciben por parte del legislador la posibilidad de reducir su carga impositiva hacia las arcas públicas de manera legal, se materializa a través de:

- Exenciones Fiscales: donde se le permite a una empresa o autónomo el impago de determinado impuesto.

- Deducciones: cuando se asimila la devolución parcial o completa de un determinado impuesto por una inversión o gasto relacionados con la actividad económica. 
- Bonificación fiscal; trata de aminorar la carga de un impuesto reduciendo o bien la base imponible o bien la cuota tributaria.

El beneficio fiscal debe recogerse expresamente en la legislación, es decir, a través de una ley, reglamento u otro. No es posible la aplicación de beneficios fiscales que no estén autorizados expresamente por el legislador. Para su aplicación es necesario el cumplimiento de requisitos específicos los cuales están sujetos a condiciones futuras y cuyo incumplimiento provocaría la reversión del beneficio otorgado.

El concepto de beneficio fiscal está estrechamente relacionado al concepto de concesión tributaria; definición que aporta un nuevo punto de vista, puesto que la intención subyacente en el concepto de gasto tributario, es la identificación de aquellas concesiones tributarias que el Estado utiliza para financiar de manera indirecta sus políticas y que, por definición, son usufructuadas por un reducido número de beneficiarios. No obstante, existen concesiones tributarias generales que financian políticas públicas, que no son catalogadas dentro de la definición de gasto tributario por el uso que de estos pueden hacer todos los contribuyentes. Un ejemplo; los ingresos exentos, las deducciones familiares y de gastos obligatorios; componentes necesarios en la estructura del gravamen.

El gasto tributario constituye un instrumento en la política pública, por ende, su eficiencia debe estar sometida a constates evaluaciones. Surge entonces una interrogante: ¿Será realmente ventajoso para el Estado?

Swift et al. (2004) mencionan los siguientes aspectos positivos de los gastos tributarios:

- Incentivan la participación del sector privado en programas económicos y sociales en que el gobierno juega un rol principal.

- Promueven la toma de decisiones del sector privado, y no tanto la del gobierno.

- Reducen la necesidad de supervisión estatal de los gastos directos equivalentes.

En este último punto, hay que precisar que si bien la aplicación de un gasto tributario reduce la necesidad de la supervisión estatal que requiere todo gasto directo al mismo tiempo, implica una supervisión y control riguroso del uso del beneficio otorgado. Así, se debería evaluar mejor la efectiva ventaja mencionada, ya que puede ocurrir que en muchos casos los costos de supervisión de los beneficios sean mayores que los gastos directos, especialmente si se contabilizan los abusos y fraudes asociados.

Sin embargo; el profesor Stanley S. Surrey, uno de los pioneros en la discusión del tema, menciona algunas desventajas de esta herramienta:

- En muchos casos, los gastos tributarios crean estímulos para que las personas hagan aquello que de todas formas habrían hecho, por lo cual el estímulo se transforma 
finalmente en una concesión innecesaria para el beneficiario. Es decir, para incentivar a uno se asume el costo de varios.

- Las administraciones tributarias no tienen suficiente experiencia en administrar y controlar programas de gasto público. Sin embargo, cuando se implementa un gasto tributario se ven obligadas a cumplir esta función y, dado que es más difícil controlar un sistema con muchas excepciones, ello puede dar lugar al fraude o incluso a mayores errores involuntarios.

- En la medida en que un gasto tributario favorece a un sector específico de la economía, produce un desvío de recursos no deseado hacia el sector favorecido. Sin embargo, esto no constituye necesariamente una desventaja en aquellos casos en que el gasto tributario busca corregir una distorsión causada previamente por el impuesto, como ocurre con los estímulos a la inversión y el ahorro.

- Para recaudar una cantidad determinada se requerirán tasas más altas, si una parte de la recaudación potencial se pierde por la aplicación de gastos tributarios obligan a mantener elevadas las tasas.

Desde el punto de vista de las políticas públicas, los gastos tributarios son una herramienta alternativa de intervención estatal, que persigue resultados similares a los que se podrían obtener mediante el gasto público directo, este está sujeto permanentemente al escrutinio público. Por lo que él gasto tributario debería estar sujeto a controles similares y criterios de transparencia que este último.

Un control menos riguroso de los gastos tributarios en relación con los gastos directos crea incentivos para establecer subsidios y transferencias en la forma de los primeros, al margen de las consideraciones objetivas que podrían justificar tal elección. Esto hace peligrar las funciones distributivas, macroeconómicas y administrativas del presupuesto y puede poner en riesgo la función primaria de recaudación de ingresos del sistema tributario.

Los presupuestos de gastos tributarios son informes financieros que dan cuenta de los problemas anteriormente señalados. En efecto, le otorgan transparencia al uso de las concesiones tributarias y facilitan el adecuado control de éstas y la asignación más eficiente de los recursos.

\section{Propuesta del Informe de Presupuesto de gasto tributario}

Tomando algunas directrices de buenas prácticas internacionales, se conforma un resumen para la estimación y presentación de informes de presupuestos de gastos tributarios: 


\section{Identificación de los gastos tributarios:}

Los gastos tributarios deberían ser identificados mediante el uso de un impuesto de referencia. La referencia no tiene que representar necesariamente la base tributaria normativa, debería ser global y única.

\section{Control presupuestario de los gastos tributarios:}

- Todos los gastos tributarios tendrían que ser estimados e integrados en la documentación sobre gastos presentada a las autoridades de presupuesto, para todos los impuestos relevantes. Los gastos directos y los gastos tributarios deberían aparecer "frente a frente" en esta documentación, para el mismo número de años.

- Tendrían que ser incluidos en los límites del gasto total, o bien habría que establecer un límite específico para ellos.

- Deberían ser revisados al igual que los gastos regulares en el proceso presupuestario anual y ser revisados por el personal de los ministerios y de la oficina de presupuestos.

- Deberían ser sometidos a procedimientos especiales de evaluación, incluyendo la revisión de programas.

- Tendrían que ser asignados a los ministerios.

\section{Estimación de los gastos tributarios:}

Los gastos tributarios se estiman por el método que sea más factible según las condiciones del país y es corregida por un margen tributario equivalente.

Podemos enunciar tres métodos para estimar el gasto tributario, según tres conceptos diferentes:

- El ingreso renunciado; mide la pérdida de ingresos que se produce después de introducir un gasto tributario. Supone que no hay cambio alguno de comportamiento en los contribuyentes, es decir, que su comportamiento es el mismo que exhibían durante la aplicación del gasto tributario. Este método es conocido también como medición ex post.

- El ingreso ganado procura estimar la recaudación adicional que es posible obtener con la derogación de un gasto tributario. A diferencia del método anterior, en éste, denominado también medición ex ante se toman en cuenta los cambios de comportamiento de los contribuyentes. En la práctica, la aplicación de este método es bastante limitada, pues plantea la dificultad de que se debe disponer de estimaciones de las elasticidades de oferta y demanda de los bienes o rentas favorecidos con un tratamiento tributario preferencial. Este método también debe considerar los cambios de comportamiento en relación con la evasión. Una parte del ingreso potencial derivado de la derogación de un gasto tributario terminará siendo 
evadida, tal como ocurre con una proporción de la recaudación de todos los impuestos.

- El gasto directo equivalente; estima el subsidio o la transferencia que dejaría a los contribuyentes con un ingreso neto de impuestos similar al que obtienen con la existencia del gasto tributario.

La responsabilidad de la estimación de los gastos tributarios tendría que recaer sobre el Ministerio de Finanzas y Precios (MFP).

En el mundo no existe un modelo de estimación del gasto tributario generalmente aceptado. El método más empleado es el expost por su facilidad de aplicación en comparación con el resto de los métodos, agiliza y facilita el proceso de cálculo, así como el análisis de los resultados que estos arrojen.

La metodología que se empleará entonces para la estimación del gasto tributario será laex post, mencionada anteriormente; la cual se basa en un contexto de ingresos perdidos por impuestos y en el empleo de análisis comparativos: horizontales, verticales y porcentuales.

La estimación del gasto tributario se presentará a través de un Informe ("Estimación del gasto tributario año 20xx") con un fundamento jurídico, que lo dotará de la legalidad necesaria para su inclusión en la Ley del Presupuesto del Estado emitida anualmente.

Para la elaboración del documento se deberá crear una comisión que engrane actores de cada una de las Instituciones de la Administración Tributaria del país: Ministerio de Finanzas y Precios (MFP), Oficina Nacional de Administración Tributaria (ONAT) y la Aduana General de la República (AGR).

Para articular objetivamente la propuesta diseñada de la presente investigación es necesario organizar la información a utilizar, de manera tal que el procedimiento sea transparente y se eviten errores en el momento de la estimación.

A continuación, se detallará la estructura del Informe:

\section{Detalles primarios:}

- Las definiciones y conceptos básicos.

- Ley que respalda dicho documento.

- Las instituciones que conforman la comisión que lo redacta.

\section{Los Impuestos:}

- Se especifican los elementos estructurales de cada uno:

- Hecho imponible

- Sujetos pasivos 
- Base imponible

- Tipo impositivo

Tabla N 1. Estimación del Gasto:

\begin{tabular}{|c|c|c|c|c|c|c|}
\hline $\begin{array}{c}\text { Impuesto } \\
(1)\end{array}$ & $\begin{array}{c}\text { Base } \\
\text { Imponible } \\
(2)\end{array}$ & $\begin{array}{c}\text { TIE } \\
(3)\end{array}$ & $\begin{array}{c}\text { Cuota } \\
\text { Tributaria } \\
\text { Especial } \\
(4)\end{array}$ & $\begin{array}{c}\text { TIN } \\
(5)\end{array}$ & $\begin{array}{c}\text { Cuota } \\
\text { Tributaria } \\
(6)\end{array}$ & $\begin{array}{c}\text { Gasto } \\
\text { Tributario } \\
(7)\end{array}$ \\
\hline & & & $2 \times 3$ & & $2 \times 5$ & $6-4$ \\
\hline
\end{tabular}

Fuente Elaboración propia

\section{Leyenda:}

- Impuestos que contengan concesiones en el sector que se analice.

- Cuantificación del hecho imponible a la que se le aplica el tipo impositivo

- TIE(Tipo Impositivo Especial): Proporción especial que se aplica sobre la base imponible con objeto de calcular el gravamen.

- Resultado de aplicar un tipo impositivo especial.

- TIN (Tipo Impositivo Normado): proporción que se aplica sobre la base imponible con objeto de calcular el gravamen.

- Cantidad que representa el gravamen.

- Lo que se deja de ganar por el beneficio fiscal otorgado.

\section{Diagnóstico:}

Se elabora el informe con el resumen de los resultados obtenidos de la estimación.

La normativa tributaria cubana ha establecido tratamientos preferenciales, incorporados a determinadas políticas de estímulo en sectores específicos: el agropecuario, el de la inversión extranjera y las zonas francas y parques industriales.

En el sector subrayado enfocaremos el ejemplo práctico que demuestra la necesidad de la estimación del gasto tributario:

A continuación, fundamentación jurídica que van a respaldar las operaciones:

\section{Impuesto sobre utilidades}

Se establece un Impuesto sobre Utilidades al que están obligadas las personas jurídicas, cubanas y extranjeras que obtengan utilidades imponibles, con independencia a su forma de organización o régimen de propiedad. 
El tipo impositivo es del treinta y cinco por ciento (35\%) sobre la utilidad imponible, el cual puede elevarse a un cincuenta $(50 \%)$ por la explotación de recursos naturales.

Las Empresas Mixtas y partes en los Contratos de Asociación Económica Internacional. Según la Ley 118 de Inversión Extranjera pagan un 0\% durante 8 años y excepcionalmente por un período superior. Posteriormente el $15 \%$ y un $0 \%$ sobre utilidades reinvertidas; manteniendo la posibilidad de elevarse a un cincuenta $(50 \%)$ por explotación de recursos naturales.

\section{Impuesto por la utilización de la fuerza de trabajo}

Se establece un Impuesto a las personas naturales y jurídicas, cubanas o extranjeras, que utilicen fuerza de trabajo remunerada.

La base imponible del impuesto lo constituye el monto total de las remuneraciones.

El tipo impositivo del Impuesto por la Utilización de la Fuerza de Trabajo disminuye de forma progresiva con la entrada en vigor de la Ley 113 del "Del Sistema Tributario", conforme a las reglas que establece el artículo 231. Para el actual año sería cinco por ciento (5\%). Según la Ley No. 118: "Ley de la Inversión Extranjera”, se exime del pago del Impuesto sobre los Ingresos Personales, a los inversionistas extranjeros socios en Empresas Mixtas o partes en Contratos de Asociación Económica Internacional, por los ingresos obtenidos a partir de los dividendos o beneficios del negocio.

\section{Impuesto sobre ventas y servicios}

Se establece un Impuesto sobre las Ventas de los bienes destinados al uso y consumo que sean objeto de compra-venta, importados o producidos, total o parcialmente en Cuba, para ser comercializados en la red mayorista y minorista, sin perjuicio de la moneda en que se opere.

Las personas jurídicas autorizadas a comercializar bienes de forma mayorista, aplican sobre el importe total de las ventas, el tipo impositivo del dos por ciento $(2 \%)$, mientras que para las autorizadas a comercializar de forma minorista, el tipo impositivo es del diez por ciento (10\%), al igual que para el Impuesto sobre los Servicios de telefonía, de comunicaciones, energía eléctrica, agua, gas, alcantarillado, transporte de carga y pasajeros, gastronómicos, así como otros servicios que se presten en el territorio nacional, con independencia de la moneda en que se ofrezcan.

Para las bebidas alcohólicas, vinos, licores y cervezas; cigarros y tabacos; hidrocarburos; artículos sanitarios; transmisión de energía eléctrica; y otros productos y servicios que se establezcan en la Ley Anual del Presupuesto; se establece un impuesto especial que se aplica en adición a los Impuestos sobre las Ventas y los Servicios. 
Las Empresas Mixtas y partes en los Contratos De Asociación Económica Internacional. Según la Ley 118 de Inversión Extranjera paga 0\% durante el primer año de operaciones, posteriormente una bonificación del $50 \%$ sobre las ventas mayoristas y sobre los servicios.

\section{Contribución Territorial para el Desarrollo Local}

Se establece una contribución para el desarrollo sostenible de los municipios, que grava los ingresos por la comercialización de bienes o prestación de servicios que obtengan las empresas, sociedades mercantiles y cooperativas, por sí mismas y por sus establecimientos en cada territorio.

La base imponible de la Contribución Territorial para el Desarrollo Localestá constituida por la totalidad de los ingresos provenientes de la venta de bienes y la prestación de servicios, atribuibles a cada establecimiento o a la propia empresa, sociedad o cooperativa cuando genere por sí misma estos ingresos.

Los contribuyentes pagan esta Contribución, aplicando el tipo impositivo que se disponga en la Ley Anual del Presupuesto. Este año está establecido un tipo impositivo del uno por ciento (1\%). Para este impuesto la Ley 118 establece que se eximen del pago durante el período de recuperación de la inversión, a las Empresas Mixtas, así como a los inversionistas nacionales y extranjeros partes en Contratos De Asociación Económica Internacional.

\section{Impuesto sobre Ingresos Personales}

Pagan este impuesto los propietarios o usufructuarios de tierra, tenedores de ganado sin tierra y productores individuales de alimentos de origen animal o vegetal. Para el pago de este impuesto, se establece un importe mínimo de un cinco por ciento (5\%) por las ventas efectuadas a entidades acopiadoras o comercializadoras, así como un pago adicional que se realizará al final del año fiscal. Según la Ley 118 se exime del pago del Impuesto Sobre Los Ingresos Personales, a los inversionistas extranjeros socios en Empresas Mixtas o partes en Contratos De Asociación Económica Internacional, por los ingresos obtenidos a partir de los dividendos o beneficios del negocio.

La estimación se realizará con datos experimentales expresados en millones de pesos en Moneda Nacional (cup). 
Tabla N 2 Sector: Inversión Extranjera

\begin{tabular}{|l|c|c|c|c|c|c|}
\hline \multicolumn{1}{|c|}{ Impuesto } & $\begin{array}{c}\text { Base } \\
\text { Imponible }\end{array}$ & TIE & $\begin{array}{c}\text { Cuota } \\
\text { Tributaria } \\
\text { Especial }\end{array}$ & TIN & $\begin{array}{c}\text { Cuota } \\
\text { Tributaria }\end{array}$ & $\begin{array}{c}\text { Gasto } \\
\text { Tributario }\end{array}$ \\
\hline Sobre utilidades & 500 & 0,15 & 75 & 0,35 & 175 & 100 \\
\hline $\begin{array}{l}\text { por la utilización de la } \\
\text { fuerza de trabajo }\end{array}$ & 350 & 0 & 0 & 0,05 & 17,5 & 17,5 \\
\hline Sobre ventas & 600 & 0,01 & 6 & 0,02 & 12 & 6 \\
\hline Sobre servicios & 550 & 0,05 & 27,5 & 0,1 & 55 & 27,5 \\
\hline $\begin{array}{l}\text { Sobre la contribución al } \\
\text { desarrollo local }\end{array}$ & 200 & 0 & 0 & 0,01 & 2 & 2 \\
\hline $\begin{array}{l}\text { Sobre ingresos } \\
\text { personales a socios o a } \\
\text { partes }\end{array}$ & 450 & 0 & 0 & 0,15 & 67,5 & 67,5 \\
\hline Total & & & $\mathbf{1 0 8 , 5}$ & & $\mathbf{3 2 9}$ & $\mathbf{2 2 0 , 5}$ \\
\hline
\end{tabular}

Fuente Elaboración propia

Cuba presenta una economía muy abierta, no es un país rico en recursos naturales y el ahorro nacional no es suficiente para hacer inversiones que requiere el país para su desarrollo económico y social; de allí que acudan a financiamiento externo y traten de incentivarlos a través de beneficios fiscales

La ley de inversión extranjera (Ley 118), se aprobó en el 2014, en aquella época las estimaciones apuntaban a 2000 millones de dólares anuales de capital extranjero para contemplar el desarrollo a mediano y largo plazo.

Hasta noviembre del 2016 se logró atraer compromisos de capital de 1300millones de dólares por lo que no se logró en esta primera etapa un avance perceptible ante el crecimiento que se necesita; el cual sería de un 5\% a un $7 \%$ del PIB. Para poder alcanzar dichas tasa de crecimiento se necesita una inversión del $27 \%$ sobre el PIB; al cual no se ha podido llegar hasta la fecha.

Analizando la razón) entre el total de gasto tributario y el total de la cuota tributaria (cuadro 1.1), se evidencia que el Estado renuncia a recaudar el $67 \%$ de lo que debería, considerándose un gasto tributario puesto que no se han conseguido los resultados que se esperaban con la asignación de beneficios fiscales en este sector. 


\section{Conclusiones}

- A partir de las leyes que otorgan concesiones fiscales en el sector de la inversión extranjera se logra percibir que no se están logrando los beneficios esperados, en pro del desarrollo económico social cubano, por lo que se transfiguran gastos tributarios que no son controlados por el Estado.

- Se propuso aplicar para la estimación el método de cálculo ex post, por ser el que más se ajusta a las condiciones del país.

- Se presenta, además, un modelo de fácil comprensión y usopara preparar el informe del presupuesto del gasto tributario.

\section{Referencias bibliográficas.}

De la Inversion Extranjera y sus avances. (2019). Granma.

Cuba, o. o. (marzo de 2018). granma. Recuperado el 13 de mayo de 2019, de granma: www.granma.cu/cuba/

Engel, E. (5 de Mayo de 2012). Gasto Tributario: que es y porque importa. Reportajes de la tercera.

Exterior, M. d. (2018). comercio Exterior. Recuperado el abril de 2019

Luis Villela, A. L. (2009). Los presupuestos de gastos tributarios.

Oscar Figueredo Reinaldo, J. R. (27 de diciembre de 2018). CUBAHORA. Recuperado el mayo de 2019, de CUBAHORA.

Triana, J. (27 de diciembre de 2018). EBM. Recuperado el mayo de 2019

Tributaria, C. I. (2017). Gasto Tributario en Republica Dominicana. Estimacion para el presupuesto general del Estado del año 2018.

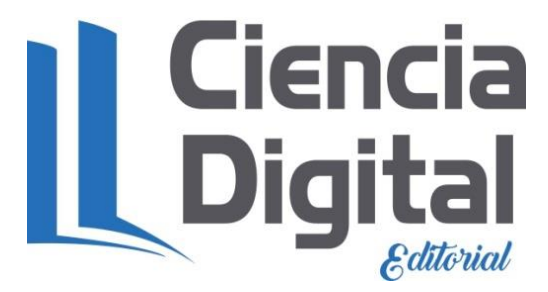


Para citar el artículo indexado.

Díaz López, E., \& Puig Morales, C. (2019). Estimación del gasto tributario en Cuba. ConcienciaDigital, 2(2), 53-65. https://doi.org/10.33262/concienciadigital.v2i1.952

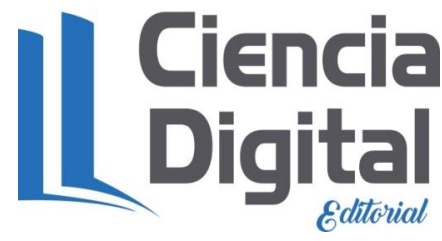

El artículo que se publica es de exclusiva responsabilidad de los autores y no necesariamente reflejan el pensamiento de la Revista Conciencia Digital.

El articulo queda en propiedad de la revista y, por tanto, su publicación parcial y/o total en otro medio tiene que ser autorizado por el director de la Revista Conciencia Digital.
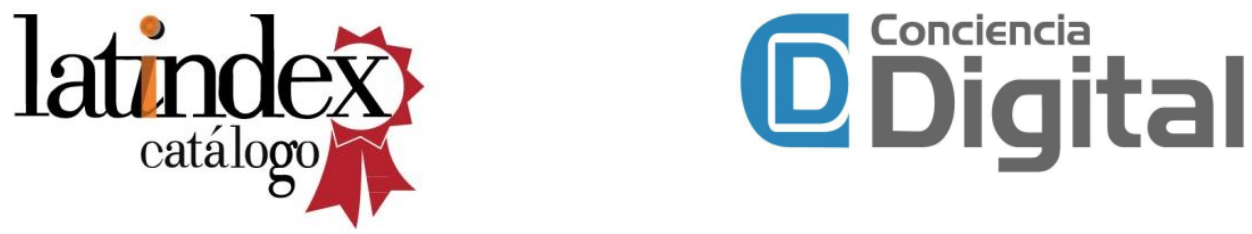